\title{
Uric Acid and Left Ventricular Hypertrophy
}

\author{
Shigeyuki Saitoh, MD
}

$\mathbf{I}$ $\mathrm{n}$ this issue of the Journal, Mitsuhashi et al report that levels of uric acid (UA) were positively associated with electrocardiographically diagnosed left ventricular hypertrophy (LVH) in healthy Japanese men! The result was independent of body mass index, hypertension, diabetes, hyperlipidemia and age; similar results were obtained in both the normal and high blood pressure (BP) subgroups. Theirs was an epidemiological study, but the reported serum concentrations of UA add important information to the assessment of risk factors and preventing cardiovascular disease, especially heart failure.

\section{Article p 667}

The relationship between UA and cardiovascular disease has been known since the first half of the $20^{\text {th }}$ century and several studies have identified an association between increased UA and cardiovascular risk in the general population. The positive association between serum UA and hypertension was also observed over a century ago. Although elevated UA levels have been predictive of hypertension in epidemiological studies, the relationship between UA and $\mathrm{BP}$ is confounded by numerous factors, including age, diabetes, obesity, alcohol use, and sodium intake or volume status. Recent findings in animal models have helped elucidate possible mechanisms whereby UA may lead to hypertension, and have spurred a renewed interest in discerning a causal role for elevated UA in hypertension.

On the other hand, the presence of hypertensive organ damage signals a condition of increased risk for cardiovascular and renal morbidity and mortality. Thus, the search for $\mathrm{LVH}$, atherosclerosis and microalbuminuria as hypertensive organ damage, which likely reflect both the severity of BP load and other nonhemodynamic risk factors, is currently recommended as part of global risk assessment. Mitsuhashi et al show new findings of a relationship between UA and LVH in Japanese, regardless of the presence of hypertension!

There are already reports of the relation between UA and LVH in Japanese with hypertension. For example, Kurata et al reported that serum UA levels correlated positively with left ventricular (LV) mass and indexed LV mass (LVMI) in male hypertensive patients, but not in female hypertensive patients in a cross-sectional study? Iwashima et al also demonstrated that UA is independently associated with LVMI

The opinions expressed in this article are not necessarily those of the editors or of the Japanese Circulation Society.

(Received February 2, 2009; accepted February 2, 2009)

Second Department of Internal Medicine, Sapporo Medical University School of Medicine, Sapporo, Japan

Mailing address: Shigeyuki Saitoh, MD, Second Department of Internal Medicine, Sapporo Medical University, S-1, W-16, Chuo-ku, Sapporo 060-8543, Japan. E-mail: ssaitoh@sapmed.ac.jp All rights are reserved to the Japanese Circulation Society. For permissions, please e-mail: cj@j-circ.or.jp and suggested that the combination of hyperuricemia and $\mathrm{LVH}$ is an independent and powerful predictor of cardiovascular disease?

With the exception of specific genetic defects in purine metabolism, increased UA is generally associated with important risk factors for atherosclerosis, such as hypertension, abdominal obesity, insulin resistance, metabolic syndrome and heart failure. Many studies have also clearly shown an association between increased UA concentrations and oxidative stress, endothelial dysfunction and inflammation. At the very least, an increased UA level is an independent marker of cardiovascular disease and a risk factor in cardiovascular diseases and hypertension. The question is whether UA is the cause of these risk factors or a morbid vascular change.

Because of being an epidemiological study, the results of Mitsuhashi et al's investigation do not suggest whether an elevation of the serum UA level is the cause or result of $\mathrm{LVH}$ ! A consideration of the mechanism of UA production and metabolism offers insight into the relationship between UA level and cardiovascular change. Primarily, the association between UA and LVH might relate to an association of UA with other risk factors, especially renal dysfunction, oxidative stress, BP, and obesity. UA is excreted primarily by the kidney, so decreased renal perfusion could lead to increased serum UA and activation of the renin-angiotensin system; angiotensin II is essential for the development of LVH by myocardial remodeling4. It is well known that angiotensin II induces hypertrophy and hyperplasia of myocytes and vascular smooth muscle cells, as well as influencing the expression of fibrogenic cytokine, and possibly inducing perivascular and interstitial fibrosis 5

Secondly, UA levels may reflect xanthine oxidase pathway activity, which has the potential to contribute to the progression of LV dysfunction by interfering with myocardial efficiency ${ }^{6}$ and myofilament calcium sensitivity? $\mathrm{UA}$ is a metabolic byproduct of purine metabolism and its serum level may increase because of increased generation, decreased excretion, or a combination of these mechanisms. UA is produced in the terminal step of purine metabolism catalyzed by xanthine oxidase (XO). XO pathway activity also results in the production of superoxide. $\mathrm{XO}$ is inhibited by allopurinol, which inhibited progression of cardiac hypertrophy in an animal model of hypertension without changing BP?

Furthermore, there are several possible contributors to increased UA production in cardiac disease, especially heart failure, including increased abundance and activity of XO, increased conversion of xanthine dehydrogenase to $\mathrm{XO}$, or increased XO substrate resulting from enhanced ATP breakdown to adenosine and hypoxanthine under such conditions. XO activity participates in both mechano-energetic uncoupling and vascular dysfunction in the failing circulation. Mechano-energetic uncoupling is the process whereby cardiac energy consumption remains the same or increases 
while cardiac work falls dramatically, and is increasingly being perceived as a potential key lesion in the failing heart.

On the other hand, there is the possibility that UA itself may induce LVH. Previous reports have shown that UA impairs NO generation and induces endothelial dysfunction and smooth muscle cell proliferation? Moreover, UA is able to induce inflammatory mediators, such as tumor necrosis factor, in vitro and potentially stimulates mitogen-activated protein kinases, which are known to induce cardiac hypertrophy $!^{10}$ Indeed, accumulating data support the idea that UA possesses specific toxic or other properties that could contribute to cardiac hypertrophy and heart failure pathophysiology. These findings reveal that UA may be the cause of cardiac hypertrophy in part, attributable to an increase in its serum level, via stimulation of endothelial dysfunction, smooth muscle cell proliferation, and inflammation.

So far there is strong evidence that increased UA is associated with atherosclerosis and an increased risk of cardiovascular events. The findings of Mitsuhashi et al. also suggest that the serum level of UA affects cardiac hypertrophy in men! However, whether UA per se is a cause of cardiovascular disease, especially cardiac hypertrophy, remains to be settled. Prospective randomized studies targeting UA reduction are necessary to finish this discussion.

This finding by Mitsuhashi et $\mathrm{al}^{1}$ is not only potentially of value in preventing cardiac hypertrophy but also raises interesting questions regarding the pathophysiological action of UA on the cardiovascular system.

\section{References}

1. Mitsuhashi H, Yatsuya H, Matsushita K, Zhang H, Otsuka R, Muramatsu T, et. al. Uric acid and left ventricular hypertrophy in Japanese men. Circ J 2009; 73: 667-672.

2. Kurata A, Shigematsu Y, Higaki J. Sex-related differences in relations of uric acid to left ventricular hypertrophy and remodeling in Japanese hypertensive patients. Hypertens Res 2005; 28: 133-139.

3. Iwashima Y, Horio T, Kamide K, Rakugi H, Ogihara T, Kawano Y. Uric acid, left ventricular mass index, and risk of cardiovascular disease in essential hypertension. Hypertension 2006; 47: 195-202.

4. Itihara S, Senbonmatsu T, Price E Jr, Ichiki T, Gaffney FA, Inagami T. Angiotensin II type 2 receptor is essential for left ventricular hypertrophy and cardiac fibrosis in chronic angiotensin II-induced hypertension. Circulation 2001; 104: 346-351.

5. Nakahara T, Tanaka Y, Hirayama Y, Asano K, Adachi H, Shiokawa $\mathrm{G}$, et al. Left ventricular hypertrophy and geometry in untreated essential hypertension is associated with blood levels of aldosterone and procollagen type III amino-terminal peptide. Circ J 2007; 71: $716-721$.

6. Cappola TP, Kass DA, Nelson GS, Berger RD, Rosas GO, Kobeissi $\mathrm{ZA}$, et al. Allopurinol improves myocardial efficiency in patients with idiopathic dilated cardiomyopathy. Circulation 2001; 104: 2407-2411.

7. Pérez NG, Gao WD, Marbán E. Novel myofilament calcium-sensitizing property of xanthine oxidase inhibitors. Circ Res 1998; 83: 423-430.

8. Laakso JT, Teravainen TL, Martelin E, Vaskonen T, Lapatto R. Renal xanthine oxidoreductase activity during development of hypertension in spontaneously hypertensive rats. J Hypertens 2004; 22: 1333-1340.

9. Rao GN, Corson MA, Berk BC. Uric acid stimulates vascular smooth muscle cell proliferation by increasing platelet-derived growth factor A-chain expression. J Biol Chem 1991; 266: 8604-8608.

10. Watanabe S, Kang DH, Feng L, Nakagawa T, Kanellis J, Lan H, et al. Uric acid, hominoid evolution, and the pathogenesis of salt-sensitivity. Hypertension 2002; 40: 355-360. 